\title{
Dietary Iron Overload Induces Visceral Adipose Tissue Insulin Resistance
}

\author{
Paola Dongiovanni, ${ }^{\star}$ Massimiliano Ruscica ${ }^{\dagger}$ Raffaela Rametta, ${ }^{*}$ Stefania Recalcati, ${ }^{\ddagger}$ Liliana Steffani, $^{\dagger}$ Stefano Gatti, ${ }^{\S}$ \\ Domenico Girelli, ${ }^{\top}$ Gaetano Cairo, ${ }^{\ddagger}$ Paolo Magni, ${ }^{\dagger}$ Silvia Fargion, ${ }^{*}$ and Luca Valenti ${ }^{* \uparrow}$
}

From the Department of Pathophysiology and Transplantation,* Centro Malattie Metaboliche del Fegato, Università degli Studi di Milano, and Internal Medicine, Fondazione IRCCS Ca' Granda Ospedale Maggiore Policlinico, Milano; the Department of Pharmacological and Biomelecular Sciences, ${ }^{\dagger}$ and the Department of Human Morphology and Biomedical Sciences Città Studi, ${ }^{\ddagger}$ Università degli Studi di Milano, Milano; the Preclinical Surgical Research Center, ${ }^{\S}$ Fondazione IRCCS Ca' Granda Ospedale Maggiore Policlinico Milano, Milano; and the Section of Internal Medicine, "Department of Medicine, University of Verona, Verona, Italy

\author{
Accepted for publication \\ February 4, 2013. \\ Address correspondence to \\ Luca Valenti, M.D., Depart- \\ ment of Pathophysiology and \\ Transplantation Università degli \\ Studi, Fondazione IRCCS Ca' \\ Granda Ospedale Maggiore \\ Policlinico, Padiglione Granelli, \\ via F Sforza 35, 20122, Milan, \\ Italy. E-mail: luca.valenti@ \\ unimi.it.
}

\begin{abstract}
Increased iron stores associated with elevated levels of the iron hormone hepcidin are a frequent feature of the metabolic syndrome. The aim of this study was to assess the effect of dietary iron supplementation on insulin resistance and the role of hepcidin in $\mathrm{C} 57 \mathrm{Bl} / 6$ male mice fed a standard or iron-enriched diet for 16 weeks. Iron supplementation increased hepatic iron and serum hepcidin fivefold and led to a $40 \%$ increase in fasting glucose due to insulin resistance, as confirmed by the insulin tolerance test, and to threefold higher levels of triglycerides. Iron supplemented mice had lower visceral adipose tissue mass estimated by epididymal fat pad, associated with iron accumulation in adipocytes. Decreased insulin signaling, evaluated by the phospho-Akt/Akt ratio, was detected in the visceral adipose tissue of iron overloaded mice, and gene expression analysis of visceral adipose tissue showed that an iron-enriched diet up-regulated iron-responsive genes and adipokines, favoring insulin resistance, whereas lipoprotein lipase was down-regulated. This resulted in hyperresistinemia and increased visceral adipose tissue expression of suppressor of cytokine signaling-3 (Socs3), a target of resistin and hepcidin implicated in insulin resistance. Acute hepcidin administration down-regulated lipoprotein lipase and up-regulated Socs 3 in visceral adipose tissue. In conclusion, we characterized a model of dysmetabolic iron overload syndrome in which an iron-enriched diet induces insulin resistance and hypertriglyceridemia and affects visceral adipose tissue metabolism by a mechanism involving hepcidin up-regulation. (Am J Pathol 2013, 182: 2254-2263; http://dx.doi.org/10.1016/ j.ajpath.2013.02.019)
\end{abstract}

The dysmetabolic iron overload syndrome (DIOS), characterized by fatty liver, increased ferritin levels, and increased body iron stores in the presence of insulin resistance (IR), is observed in $15 \%$ to $30 \%$ of patients with the metabolic syndrome (MetS) ${ }^{1}$ and is associated with increased risk of type 2 diabetes (T2D) and organ damage. ${ }^{2}$ Dietary iron intake has been associated with $\mathrm{T} 2 \mathrm{D},{ }^{3}$ and iron depletion decreased IR in controlled studies. ${ }^{4}$ Differently from genetic iron overload disorders, ${ }^{5}$ DIOS is characterized by preserved upregulation of the iron hormone hepcidin, ${ }^{1}$ which inhibits iron absorption and recycling by binding and inactivating the cellular iron exporter ferroportin-1.6 ${ }^{6}$ Therefore, the pathogenesis of iron accumulation in DIOS has been related to altered iron trafficking associated with steatosis, hepatic inflammation, and IR. ${ }^{1,7}$

DIOS is also associated with altered release of adipokines, ${ }^{8}$ iron treatment decreases insulin-stimulated glucose transport and increased lipolysis in adipocytes in vitro, ${ }^{9}$ and increased ferritin levels and iron indices have been associated with adipose tissue insulin resistance in a population study. ${ }^{10}$

Supported by grants from Bando Giovani Ricercatori Ricerca Finalizzata 2007, the Ministero della Salute e delle Politiche Sociali (grant GR-2007683265 to L.V.), and an Italian Ministry of University and Research grant (200989KXFN to D.G.). 
In obesity models, iron accumulation induced IR by still unclear mechanisms, whereas dietary restriction or chelation protected against diabetes and decreased IR. ${ }^{11-15}$ Mice with iron overload caused by down-regulation of hepcidin due to deletion of the hemochromatosis gene ( $\mathrm{Hfe}$ ) are characterized by decreased glucose oxidation in skeletal muscle despite increased uptake ${ }^{11}$ leading to higher hepatic glucose output but not to hyperglycemia or dyslipidemia. ${ }^{12} \mathrm{Re}-$ cently, increased hepcidin levels have been associated with MetS in the general population, ${ }^{16}$ but the role played by hepcidin in the pathogenesis of metabolic abnormalities could not be investigated in previous experimental models, which, unlike DIOS, were not characterized by increased hepcidin.

Therefore, the aims of this study were to assess whether dietary iron overload leading to increased hepcidin release influenced glucose and lipid metabolism and insulin resistance in C57Bl/6 mice and to investigate the mechanisms by analyzing insulin signaling alterations in target tissues and the role of hepcidin.

\section{Materials and Methods}

\section{Animal Model}

Six-week-old, male C57Bl/6 mice were purchased from Charles River (Charles River, Calco, Italy), housed at constant room temperature $\left(23^{\circ} \mathrm{C}\right)$ under 12-hour light/dark cycles with ad libitum access to water in compliance with the Principles of Laboratory Animal Care (NIH publication 86-23), and fed either standard iron concentration diet $(8 \mathrm{mg} / \mathrm{kg}$; control, measured by atomic absorption spectrometry in regular commercial chow diet) or an iron-enriched diet (IED; $3 \%$ carbonyl-iron, a highly pure form of iron, which does not contain carbohydrates) ${ }^{17}$ for 16 weeks. Experiments were conducted in 15 mice per group, unless otherwise specified. Body weight and food intake were measured weekly. In a second set of experiments, supplemental fructose was administered as $30 \%$ fructose in drinking water [high-fructose $\operatorname{diet}\left(\right.$ HFRD)], as a model of fatty liver. ${ }^{18}$

A glucose tolerance test (GTT; $2 \mathrm{~g} / \mathrm{kg}$ of glucose) ${ }^{19}$ and an insulin tolerance test (ITT; $0.75 \mathrm{U} / \mathrm{kg})^{20}$ were performed after prolonged overnight fasting at 16 weeks; afterward animals were anesthetized and sacrificed. For hepcidin treatment, 8 -week-old male mice $(n=5)$ fed control diet were injected i.p. with $20 \mu \mathrm{g}$ of hepcidin- 25 in $0.3 \mathrm{~mL}$ of PBS, with a second dose injected 20 hours later, ${ }^{21}$ and sacrificed at 24 hours. A comparable control group $(n=5)$ was injected with PBS only.

Blood was collected, and liver and epididymal visceral adipose tissue (VAT) were dissected and flash frozen in liquid nitrogen. Samples of liver and VAT were also collected for quantification of iron by atomic absorption spectrometry and histologic analysis. The experimental protocol was approved by the University of Milan and Italian Ministry of Health review boards (protocol 13/10).

\section{Biochemical Measurements}

Blood glucose was measured by an Accu-Check glucometer (Roche Diagnostics AVIVA, Mannheim, Germany). Serum iron, plasma triglycerides, and alanine aminotransferases levels were measured with an automated analyzer. Plasma insulin, resistin, tumor necrosis factor- $\alpha$, and IL- 6 levels were determined using a multiplex assay (kit MADPK-71K; Millipore, Billerica, MA). Measurement of lipid peroxidation (thiobarbituric acid reactive substances) was performed using the thiobarbituric acid reaction as previously described. ${ }^{22}$ Total iron regulatory protein (IRP) activity, reflecting cytosolic iron availability (the higher IRP activity, the lower iron availability), was measured by RNA band shift assay, as described. ${ }^{23}$

Serum hepcidin was measured by mass spectrometry using a method based on surface-enhanced laser desorption/ ionization time of flight. A synthetic hepcidin analog (Hepcidin 24; Peptides International, Louisville, KY) was used as an internal standard. ${ }^{24}$

\section{Isolation of Tissue RNA and qPCR}

RNA was isolated from tissues by the Trizol reagent (Life Technologies, Carlsbad, CA). First-strand cDNA was synthesized starting from $1 \mu \mathrm{g}$ of total RNA using the VILO random examers synthesis system (Life Technologies). Realtime quantitative PCR (qPCR) was performed by using the SYBR green chemistry (Fast SYBR Green Master Mix; Life Technologies). All of the reactions were delivered in triplicate with the 7500Fast Real-Time PCR system (Life Technologies) in a $25-\mu \mathrm{L}$ final volume. Primers are listed in Table 1. Custom Taqman array plates were purchased from Life Technologies, and the reactions were delivered in a $10-\mu \mathrm{L}$ final volume. Evaluated target and control genes are presented in Supplemental Table S1.

\section{Western Blot Analysis}

Cell extracts were prepared from $20 \mathrm{mg}$ of tissue, and equal amounts of proteins $(50 \mu \mathrm{g})$ were separated by SDS-PAGE and transferred to nitrocellulose membrane (BioRad, Hercules, CA). Membranes were incubated with polyclonal rabbit Akt antibody (C\#9272; Cell Signaling Technology, Danvers, MA), polyclonal rabbit phospho(p)-Akt antibody (\#9271; Cell Signaling), polyclonal rabbit Srebp1c antibody (sc-366; Santa Cruz Biotechnology, Santa Cruz, CA), polyclonal rabbit C/EBP $\alpha$ antibody (sc-61; Santa Cruz Biotechnology), and polyclonal goat $\beta$-actin antibody (sc-1615; Santa Cruz Biotechnology).

\section{Histological Analysis}

Liver and VAT tissue samples were fixed in 10\% PBSbuffered formalin. All tissues were embedded in paraffin within 48 hours of formalin fixation. Histologic staining was performed using H\&E and Perls Prussian blue method to 
Table 1 Primers for qPCR

\begin{tabular}{|c|c|c|}
\hline Gene & Forward primer & Reverse primer \\
\hline Acac & $5^{\prime}$-GCCTCTTCCTGACAAACGAG-3' & 5'-TGACTGCCGAAACATCTCTG-3' \\
\hline AdipoQ & 5'-GTTGCAAGCTCTCCTGTTCC-3' & $5^{\prime}$-CCAAGAAGACCTGCATCTCC-3' \\
\hline Chop & $5^{\prime}-\mathrm{CCTGAAAGCAGAACCTGGTC-3^{ \prime }}$ & 5'-TGATTCTTCСTCTTCGTTTCC-3' \\
\hline Fasn & 5'-AAGGCTGGGCTCTATGGATT-3' & 5'-TGAGGCTGGGTTGATACCTC-3' \\
\hline Ferritin $\mathrm{H}$ & 5'-CGAGATGATGTGGCTCTGAA-3' & 5'-GTGCACACTCCATTGCATTC-3' \\
\hline InsR & $5^{\prime}$-AAAGTTTGCCCAACCATCTG-3' & 5'-TCTCACCTTCGAGGATTTGG-3' \\
\hline Irs1 & $5^{\prime}$-CCAGCCTGGCTATTTAGCTG-3' & $5^{\prime}-\mathrm{CCCAACTCAACTCCACCACT-3^{ \prime }}$ \\
\hline Irs2 & 5'-GTAGTTCAGGTCGCCTCTGC-3' & 5'-CAGCTATTGGGACCACCACT-3' \\
\hline$L p l$ & 5'-TGGACGGTAACGGGAATG-3' & 5'-CAGCTGGATCCAAACCAGTAA-3' \\
\hline MitoNEET & $5^{\prime}$-TGCGAGTTGAGTGGATCG-3' & $5^{\prime}$-CATCGCAGAAGGGGAACTT- $3^{\prime}$ \\
\hline Sod2 & 5'-TCTGGCCAAGGGAGATGTTA-3' & 5'-CСТCCAGCAACTCTCСТTTG-3' \\
\hline Srebp-1c & $5^{\prime}$-GATCAAAGAGGAGCCAGTGC-3' & 5'-TAGATGGTGGCTGCTGAGTG-3' \\
\hline$V h L$ & $5^{\prime}$-CCACAGCTACCGAGGTCATC-3' & $5^{\prime}$-ATACACTGGCAATGTGATGTTG-3' \\
\hline Visfatin & $5^{\prime}$-ACATCCTTGAGAAATACGATGG-3' & 5'-TGGAATTTGTGGCCACTGTA-3' \\
\hline $18 S$ & $5^{\prime}-\mathrm{TTGACGGAAGGGCACCACCAG-3^{ \prime }}$ & $5^{\prime}$-GCACCACCACCCACGGAATCG-3' \\
\hline
\end{tabular}

detect iron deposits. VAT formalin-fixed, paraffin-embedded sections were stained for immunoreactive fatty acid binding protein 4 (Fabp4) using Mouse Fabp4 Antigen Affinitypurified Polyclonal Antibody (R\&D Systems, Minneapolis, $\mathrm{MN}$ ) and F4/80 (Santa Cruz Biotechnology). Tissue was stained using the anti-goat HRP-DAB Cell \& Tissue Staining Kit (R\&D Systems, Minneapolis, MN).

\section{Statistical Analysis}

Data are expressed as means $\pm \mathrm{SD}$, and data compared by analysis of variance (ANOVA) or repeated-measures ANOVA, when appropriate. Nonnormally distributed variables were log-transformed before analysis. Differences were considered significant for $P<.05$ (2-tailed).

\section{Results}

\section{IED Induces Hepatic Iron Overload}

At week 16, serum iron and hepatic iron content were higher in IED mice than in controls, whereas blood hematocrit was not different (Table 2). Hepatic hepcidin mRNA levels were increased, and serum hepcidin was also approximately fivefold higher in IED mice compared with controls (Table 2). Iron staining showed no iron depots in the liver of control mice, whereas hepatocellular and nonparenchymal (Kupffer cells) siderosis was observed in IED mice after 8 and 16 weeks (Supplemental Figure S1, A-C). Consistent with hepatic iron overload, total IRP binding activity was lower in IED mice than in controls (Supplemental Figure S1D). Liver thiobarbituric acid reactive substances, an index of lipoperoxidation, were sevenfold higher in IED mice than in controls $(P<0.0001)$ (Supplemental Figure S1E).

\section{IED Induces IR Associated with Decreased Visceral Adipose Tissue}

IED mice gained less weight than controls (Figure 1A) despite similar body length and food intake (Table 2). This finding was associated with reduced VAT mass and a reduction in the mean size of VAT adipocytes (Table 2), whereas no significant changes were observed in subcutaneous adipose tissue (SAT) (not shown). Higher fasting glucose level was observed in IED mice than in controls after 8 weeks, and the difference was maintained during the treatment period (Figure 1B). Liver histologic analysis revealed no inflammation and fibrosis (Supplemental Figure S1, A-C), and levels of alanine aminotransferase and tumor necrosis factor- $\alpha$ were not elevated in IED mice (Table 2).

Fasting serum insulin was not significantly higher, the homeostasis model assessment IR index was increased, and serum glucagon levels were not different in IED mice compared with controls (Table 2). IR was confirmed by ITT, demonstrating increased glucose levels at each time point in IED mice compared with controls (Figure 1C). Glucose levels remained higher in IED mice at the end of the GTT, but there was a lower increase during the first phase (Figure 1D).

To assess the interaction between iron overload and a lipogenic diet, experiments were replicated in mice supplemented with HFRD. In HFRD mice, IED increased hepatic iron content (Table 2), but it was not associated with 
Table 2 Characterization of C57Bl/6 Mice Fed Either Control or IED and of Mice with HFRD Supplementation

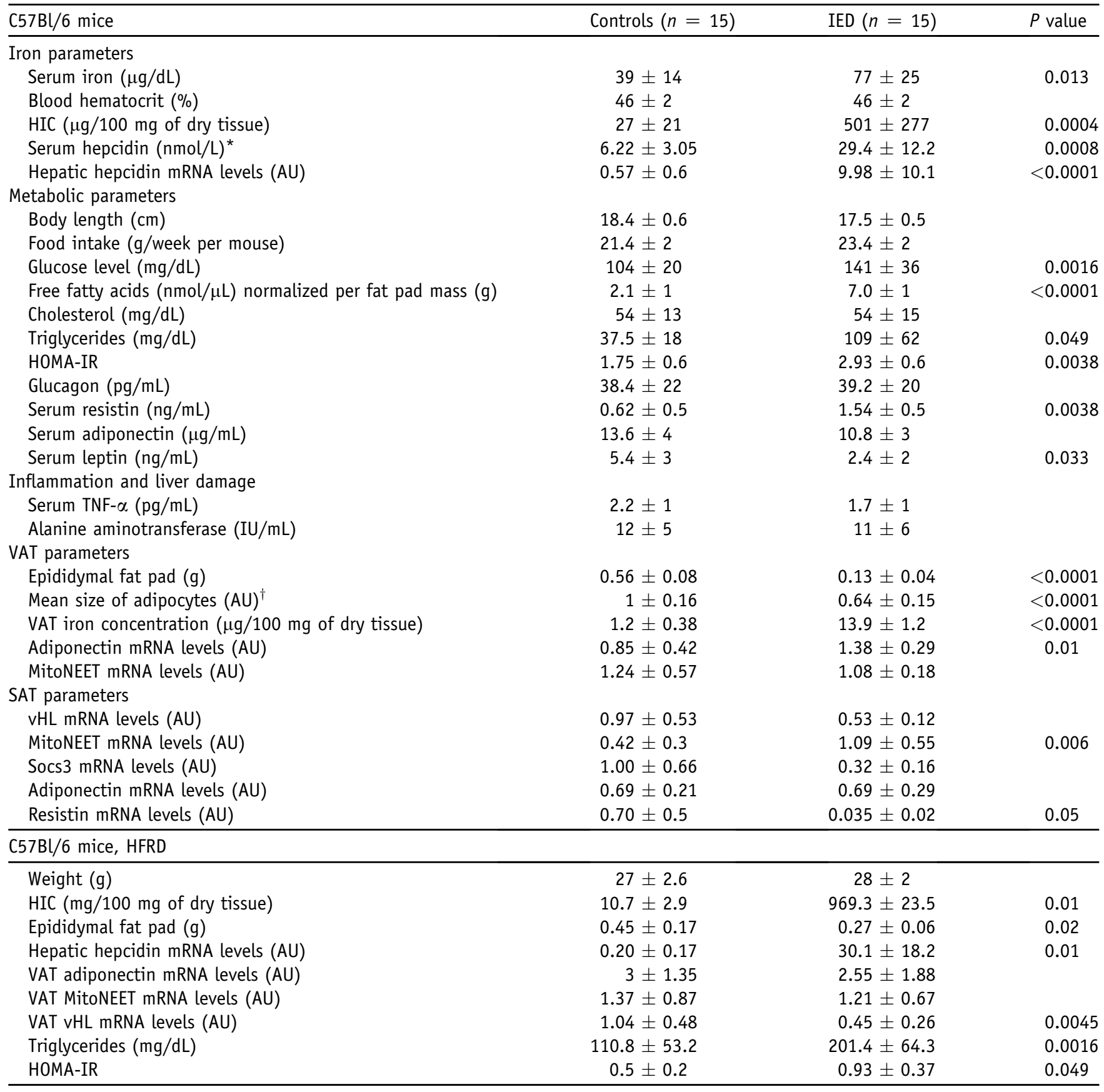

Data were collected 16 weeks from the start of the experiment (mice 20 weeks of age). Blank cells in the $P$ value column indicate lack of statistical significance.

${ }^{*}$ Available in five mice per group.

${ }^{\dagger} \mathrm{A} 35 \%$ decrease in adipocytes mean size was also detected in SAT of IED versus control mice $(P<0.0001)$.

AU, arbitrary units; HIC, hepatic iron concentration; VAT, visceral adipose tissue; vHL, von Hippel-Lindau.

decreased body weight, even if it still determined a reduction in epididymal fat pad, although to a reduced degree than in mice not supplemented with high fructose. Notwithstanding, IED increased IR by approximately twofold even in mice supplemented with HFRD, as indicated by HOMA-IR (Table 2), suggesting that IED reduces VAT mass and induces IR independently of the effect on whole body mass.

\section{IED Induces Hypertriglyceridemia}

Serum triglycerides, but not cholesterol levels, were higher in IED mice than in controls (Table 2), even when diet was supplemented by HFRD, which induces lipogenesis. IED increased hepatic mRNA levels $(P<0.0001)$ and activated protein levels $(P=0.0002)$ of Srebp-1c (cleaved nuclear form of $68 \mathrm{kDa}$ ) compared with control diet (Figure 2, A-C) 

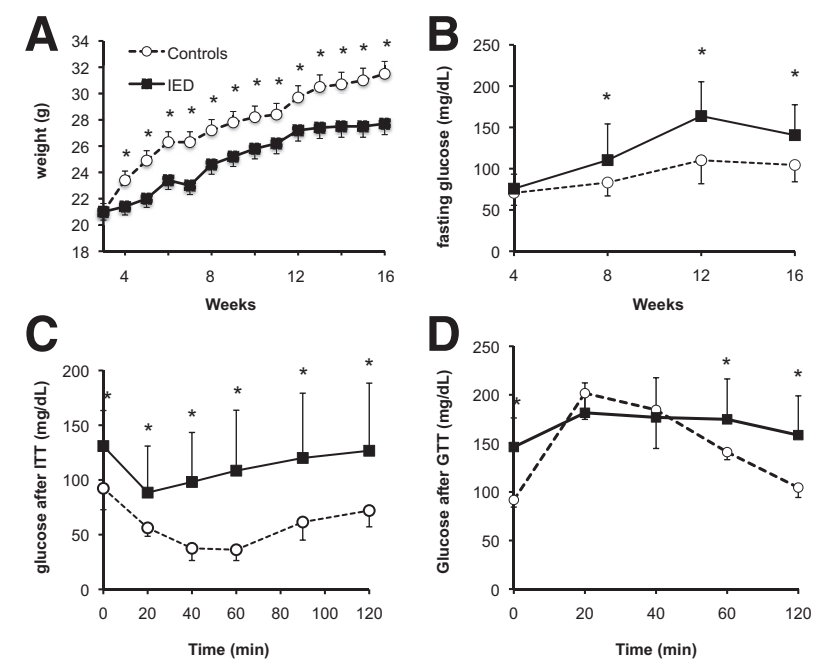

Figure 1 Effect of IED on body mass, glucose metabolism, and insulin resistance. A: Body weight changes in control and IED mice. B: Glucose levels measured after overnight fasting during treatment in IED and control mice. C: Results of the $0.75-\mathrm{U} / \mathrm{kg}$ i.p. ITT, performed at week 16 after overnight fasting, absolute glucose values. D: Results of i.p. ITT, performed at week 16 after overnight fasting, relative values. E: Results of the 2-g/kg i.p. glucose tolerance test (GTT), performed at week 16 after overnight fasting, absolute glucose values. The open circles are the controls; squares are the IED mice. Fifteen mice per group were analyzed. Data are expressed as means \pm SD. ${ }^{*} P<0.05$ versus controls at ANOVA or repeated-measures ANOVA, when appropriate. Results are representative of three independent experiments.

and induced the Srebp target ApoC3 $(P<0.0001)$, a very low-density lipoprotein apolipoprotein synthesized by the liver that inhibits lipoprotein lipase (Lpl) and hepatic lipase, thus reducing triglyceride catabolism (Figure 2A).

\section{IED Is Associated with Decreased Insulin Signaling and Altered Iron Metabolism in VAT}

IED did not influence hepatic mRNA levels of insulin receptor, insulin receptor substrate (Irs) 1, and Irs2 (not shown), whereas mRNA levels of phosphoenolpyruvatecarboxykinase and glucose-6-phosphatase were decreased in IED mice (Figure 3B). The pAkt/Akt ratio was not affected by IED in liver, skeletal muscle, and SAT, but Akt activity was down-regulated in VAT (Figure 3, A and C).

There was no difference in mRNA levels of insulin receptor, Irs1, Irs2, and glucose transporter 4 in muscle, SAT, and VAT (not shown).

Iron accumulation was detected by Perls staining in VAT of IED (both in adipocytes and macrophages) but not of control mice (Figure 4, A-D). Iron concentration $>10$-fold higher in VAT of IED mice than in controls (Table 2). There was no significant up-regulation of the number of macrophages (F4/80 positive cells) in both the VAT and SAT of IED versus control mice (not shown).

\section{Effect of IED on Gene Expression in VAT}

The mRNA levels of genes involved in iron metabolism, oxidative stress/unfolded protein response, adipogenesis/ adipose tissue metabolism, and inflammation were analyzed by the adipocytes iron plate in the VAT of IED mice and controls (Table 3). IED influenced genes regulating iron metabolism (L-ferritin and hepcidin), hypoxia signaling (von Hippel-Lindau protein), oxidative stress (Sod2), endoplasmic reticulum stress response (Xbp-1 and Chop), and antioxidant enzymes. Even if it down-regulated some cytokines and leptin, IED up-regulated resistin. Adiponectin expression was significantly higher in the VAT of IED mice compared with controls (Table 3). Notably, Lpl, mediating the uptake of lipid from the circulation, was also downregulated in VAT of IED mice but not in the liver and muscle (not shown). Generally, despite IR, decreased VAT mass, and reduced adipocyte size, genes involved in adipogenesis and lipid synthesis were up-regulated by IED.

Overexpression of resistin in VAT of IED mice was associated with higher resistin serum levels (Table 2) and with induction of mRNA levels of suppressor of cytokine signaling-3 (Socs3) (Table 3), a target of both resistin ${ }^{25}$ and hepcidin, ${ }^{26}$ and an inhibitor of insulin signaling, which would be predicted to increase VAT-IR. ${ }^{27}$ No significant up-regulation of Socs 3 mRNA levels was also observed in the liver, muscle, and SAT of IED mice versus controls (not shown).

To evaluate whether altered mitochondrial iron metabolism may be involved in the pathogenesis of IR in the VAT, we measured the mRNA expression of MitoNEET. MitoNEET is a target of glitazones expressed on the outer mitochondrial membrane, which has recently been reported to regulate mitochondrial iron metabolism and to influence SAT and IR. ${ }^{28}$ In the SAT, IED was associated with higher MitoNEET mRNA levels than in the control diet (Table 2), which is consistent with previous data ${ }^{28}$ and with downregulation of the redox-sensitive von Hippel Lindau protein as in VAT but with lower resistin and no changes in Socs3 and adiponectin mRNA levels (Table 2). However, no significant difference in MitoNEET expression was observed between IED and control diet in the VAT, despite increased expression of the mitochondrial antioxidant enzyme Sod2 (Tables 2 and 3). In addition, mRNA levels of MitoNEET, as well as of adiponectin, were not influenced by IED also in the VAT of HFRD mice. Therefore, we can assume that the effect of iron on VAT function is not mediated by altered mitoNEET expression.

\section{Acute Effect of Hepcidin on VAT Gene Expression}

As expected, acute i.p. hepcidin administration decreased serum iron (Figure 5A) and down-regulated VAT transferrin receptor expression, suggesting that this effectively increased cytoplasmic bioavailable iron within VAT, without affecting ferritin and MitoNEET mRNA levels (Figure 5B). However, short-term hepcidin treatment did not affect serum triglyceride levels (Figure 5C), fasting glucose, and i.p. ITT (Figure 5D). To dissect the relative contribution of iron overload and increased hepcidin in the alteration of VAT in 


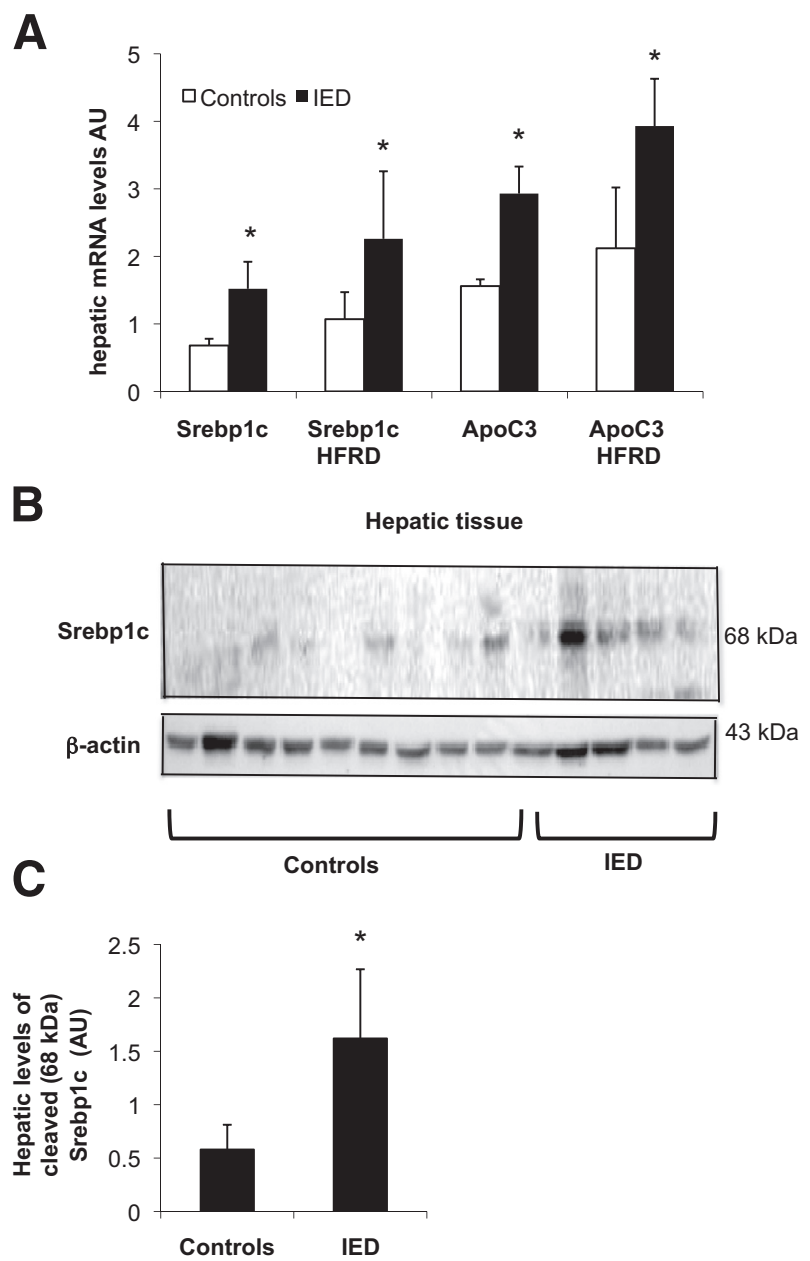

Figure 2 Effect of IED on lipid metabolism. A: Hepatic Srebp-1c and ApoC3 mRNA levels in mice fed IED or the control diet, either supplemented or nonsupplemented with HFRD. B: SREBP-1c protein levels evaluated by Western blotting. C: Densitometric analysis in control and IED mice; $\beta$-actin is shown as the loading control. Fifteen mice per group were considered. Values are expressed as means $\pm \mathrm{SD}$. $\mathrm{AU}$, arbitrary units. ${ }^{*} P<0.005$ versus controls.

IED mice, we compared the expression of genes involved in the regulation of lipid metabolism and insulin signaling, previously shown to be regulated by IED, in mice treated with hepcidin and controls. Acute hepcidin treatment was associated with down-regulation of $\mathrm{Lpl}$ and up-regulation of Socs 3 mRNA levels in VAT, whereas resistin expression was unchanged (Figure 5E). Consistent with glucose and lipid metabolism status, the activation of the insulin signaling pathway, as indicated by the pAkt/Akt ratio, was not downregulated in the VAT by acute hepcidin treatment (not shown).

\section{Discussion}

Increased body iron stores and dietary iron intake have been associated with IR, MetS, and T2D, ${ }^{1,3}$ but the role of iron in the pathophysiology of these syndromes is still debated. In this article, we analyzed whether dietary iron overload induced IR in C57Bl/6 mice and investigated the role of hepcidin. Supporting the validity of our model, iron parameters achieved with IED were comparable with those of typical patients with DIOS. ${ }^{1}$ Importantly, the preserved upregulation of hepcidin by iron overload ${ }^{1}$ allowed us to test the effect of this hormone on the regulation of glucose and lipid metabolism, which was not possible in previous studies based on genetic models characterized by a defect in the regulation of hepcidin, in which unchanged fasting glucose and triglyceride levels were observed. ${ }^{12}$

Consistent with the experimental hypothesis, IED-fed mice developed a $40 \%$ increase in glucose levels, which was not explained by loss of insulin secretion, increased glucagon, liver damage, serum levels of proinflammatory cytokines, greater food intake, or weight gain. Furthermore, hepatic insulin resistance did not contribute to hyperglycemia in IED

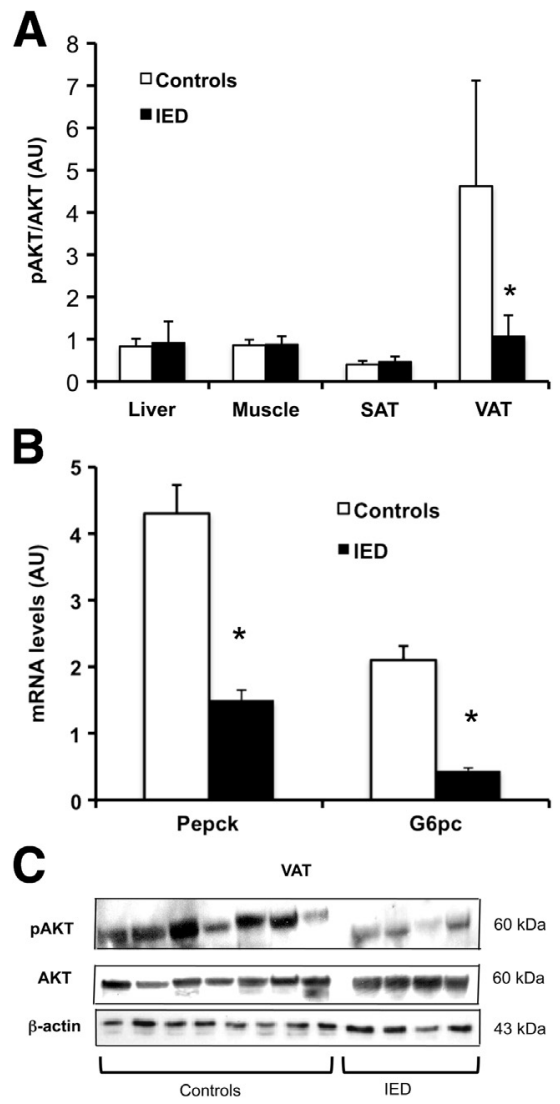

Figure 3 Evaluation of insulin resistance at different sites in C57BL/6 mice fed IED or the control diet. A: pAkt/Akt ratio, an index of activation of the insulin signaling pathway, in the liver, skeletal muscle (soleus), SAT (flank region), and VAT (epididymal) during fasting, evaluated by Western blotting (using Cell Signaling antibodies) and densitometric analysis, in controls and IED mice (15 mice per group). Image acquisition was performed by the Molecular imager Chemi doc XRS Imaging system and protein expression quantified by proprietary software (BioRad). B: Hepatic phosphoenolpyruvate-carboxykinase (Pepck) and glucose-6-phosphatase (G6pc) mRNA levels evaluated by qPCR in IED and control mice (15 mice per group). C: Levels of pAkt and Akt in VAT of IED and control mice, as assessed by Western blotting as described in $\mathbf{A}$; $\beta$-actin is shown as loading control. The figure is representative of results obtained in 15 mice per group in two independent experiments. Values are expressed as means \pm SD. AU, arbitrary units. ${ }^{*} P<0.05$ versus controls at ANOVA. 


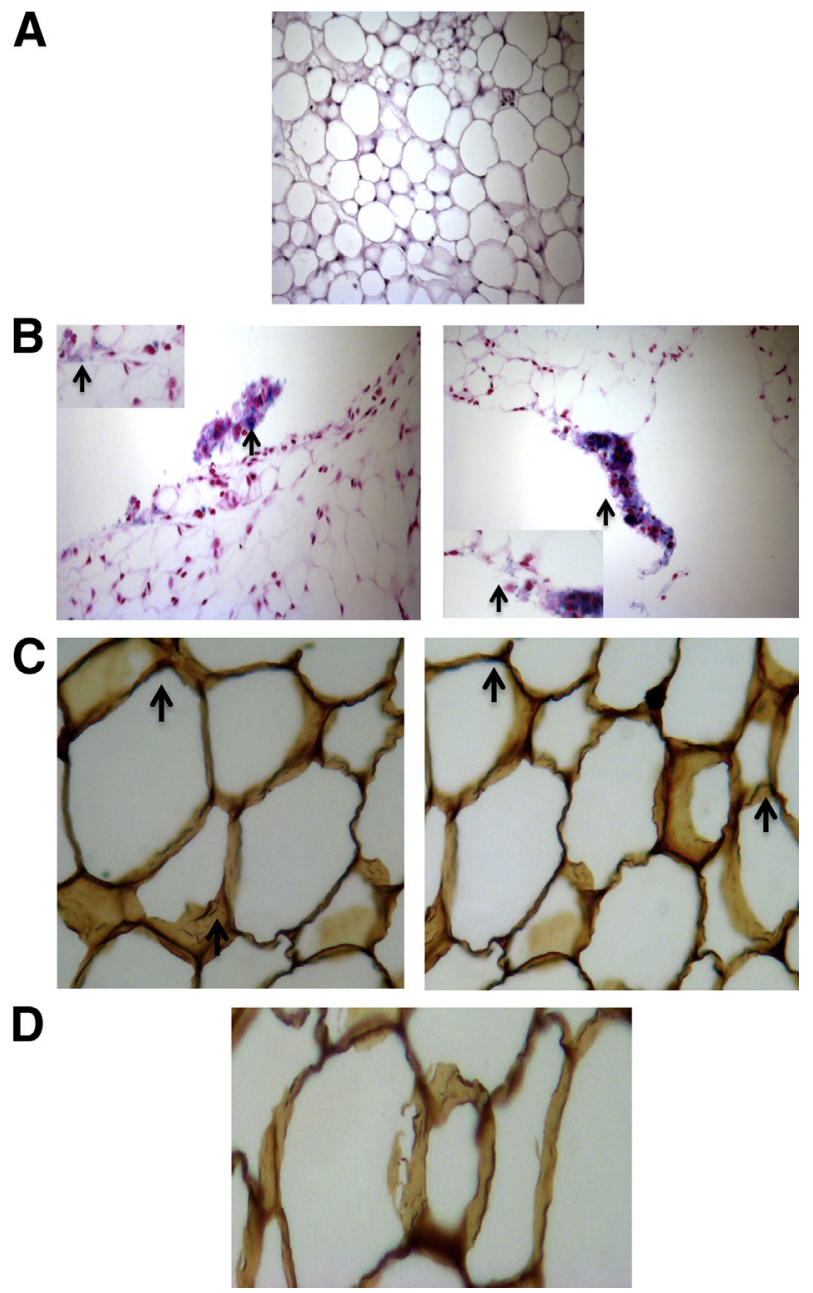

Figure 4 Iron staining of visceral adipose tissue (VAT) of IED and control mice at 16 weeks. Sections were stained by HE and Perls staining. Iron deposits are visualized as blue. Mice fed the control diet (A) and IED mice (B). Additional areas of Perls staining are highlighted in the insets. Double staining with Perls staining for iron (blue) and immunohistochemical assay with antiFabp4 antibodies (brown) in IED mice (C) and control mice (D). Iron deposits are indicated by arrows. VAT tissue samples were fixed in 10\% PBS-buffered formalin. Histologic staining was performed using H\&E and Perls Prussian blue method to detect iron deposits. VAT formalin-fixed, paraffin-embedded sections were stained for immunoreactive Fabp4 using Mouse Fabp4 Antigen Affinity-purified Polyclonal Antibody (R\&D Systems). Tissue was stained using the anti-goat HRP-DAB Cell \& Tissue Staining Kit (R\&D Systems). Figures are representative of results obtained in 15 mice per group. Original magnification: 10.

mice because the expression of gluconeogenic genes was down-regulated compared with controls, possibly as a consequence of increased insulin levels and/or reduced adipose tissue mass. Instead, mild hyperglycemia was apparently related to IR, as detected by HOMA-IR and ITT. The reduced up-regulation of glucose during GTT may be explained by the previously reported increased glucose uptake despite reduced use in mice with iron overload ${ }^{12}$ together with downregulation of gluconeogenesis in IED mice. Increased expression of Srebp1c and ApoC3 in IED mice likely represents a consequence of iron-induced endoplasmic reticulum stress and is consistent with previous reports of increased lipogenesis associated with iron accumulation, ${ }^{29}$ contributing to explain the higher triglyceride levels in IED mice.

Unlike most models of MetS, IR in IED mice was associated with decreased VAT mass, as estimated by epididymal fat pad, which in the HFRD was independent of total body mass, consistent with the clinical association of iron overload with severe IR despite less severe overweight in patients with MetS. ${ }^{1,2,30}$

To better understand the mechanisms of IED-associated IR, we analyzed the phosphorylation of $\mathrm{Akt}^{31}$ and observed a decrease of Akt activation in VAT of IED mice. Because insulin signaling is involved in the differentiation and growth of adipocytes, VAT-IR is consistent with decreased VAT mass and smaller size of adipocytes ${ }^{32}$ because reduced ability of adipocytes to store excess lipids has been associated with IR in mice ${ }^{33}$ and obese patients. ${ }^{34}$

The novel observation that IED mice developed VAT iron overload supports the possibility that iron directly

Table 3 Genes Included in the Adipocytes Iron Plate Significantly Affected by IED in the VAT of C57/Bl6 Mice

\begin{tabular}{|c|c|c|}
\hline $\begin{array}{l}\text { VAT gene expression by } \\
\text { adipocytes iron plate }\end{array}$ & $\begin{array}{l}\text { Fold increase in the } \\
\text { IED versus control } \\
\text { groups ( } n=10 \\
\text { per group) }\end{array}$ & $P$ value \\
\hline \multicolumn{3}{|l|}{ Adipogenesis } \\
\hline Stat5 & 2.03 & 0.03 \\
\hline$P G C 1-\beta$ & 1.76 & 0.03 \\
\hline$P G C 1-\alpha$ & 2.29 & 0.002 \\
\hline \multicolumn{3}{|l|}{ Inflammation } \\
\hline Ccr2 & 0.4 & 0.03 \\
\hline $\mathrm{Ccl5}$ & 0.35 & 0.03 \\
\hline Ccl2 & 0.32 & 0.008 \\
\hline \multicolumn{3}{|l|}{ Signaling } \\
\hline Akt1 & 1.63 & 0.01 \\
\hline Socs 3 & 2.1 & 0.001 \\
\hline \multicolumn{3}{|l|}{$\begin{array}{l}\text { Iron metabolism and oxidative } \\
\text { stress }\end{array}$} \\
\hline Ferritin & 2.4 & 0.0012 \\
\hline von Hippel-Lindau protein & 0.47 & 0.02 \\
\hline Hepcidin & 175.7 & 0.03 \\
\hline Catalase & 2.26 & 0.047 \\
\hline Sod2 & 1.55 & 0.05 \\
\hline Sirt2 & 1.67 & 0.031 \\
\hline Xbp1 & 1.89 & 0.003 \\
\hline Chop & 1.3 & 0.003 \\
\hline \multicolumn{3}{|c|}{ Lipid metabolism and adipokines } \\
\hline Lipoprotein lipase & 0.42 & 0.03 \\
\hline $\begin{array}{l}\text { Stearoyl-coenzyme A } \\
\text { desaturase } 1\end{array}$ & 2.02 & 0.034 \\
\hline Acetyl-CoA carboxylase & 3.5 & 0.002 \\
\hline Leptin & 0.33 & 0.05 \\
\hline Resistin & 3.3 & 0.007 \\
\hline Visfatin & 1.6 & 0.04 \\
\hline Adiponectin & 1.6 & 0.01 \\
\hline
\end{tabular}

The whole set of genes analyzed and gene expression data are presented in Supplemental Table S1.

VAT, visceral adipose tissue. 


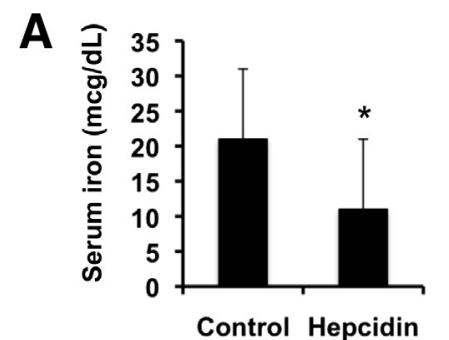

B
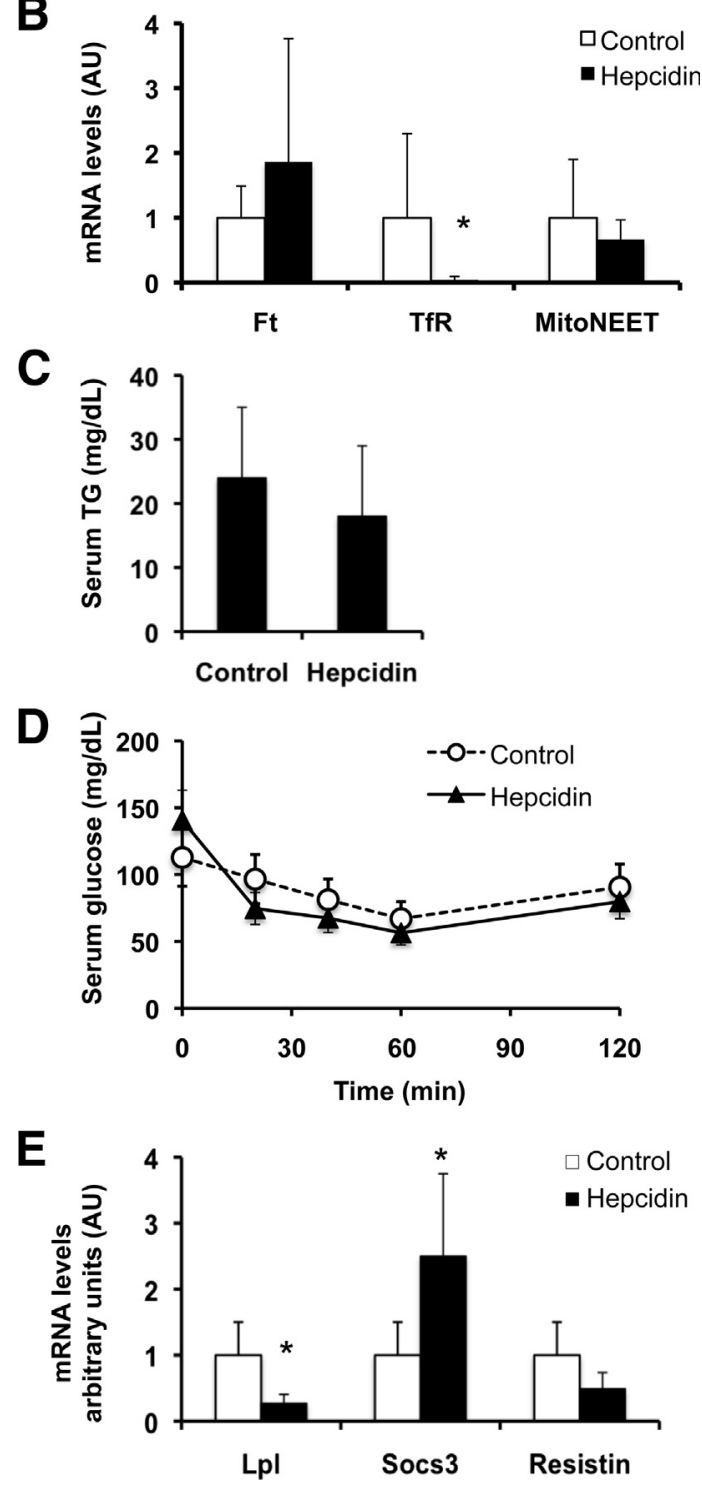

Figure 5 Effect of acute i.p. hepcidin administration on serum iron, lipid, and glucose levels and gene expression in visceral adipose tissue (VAT). Overnight fasted mice were injected i.p. with $20 \mu \mathrm{g}$ of hepcidin- 25 in $0.3 \mathrm{~mL}$ of PBS or vehicle alone, with a second dose injected 20 hours later, and sacrificed at 24 hours. Serum iron (A) and mRNA expression (B) of genes reflecting iron status; ferritin $\mathrm{H}$, transferrin receptor, and MitoNEET, evaluated by $\mathrm{qPCR}$, in the VAT of hepcidin or vehicle treated mice. C: Serum triglyceride levels at 24 hours after hepcidin injection. $\mathbf{D}$ : Insulin resistance as determined by the $0.75-\mathrm{U} / \mathrm{kg}$ i.p. ITT performed in 24 hepcidin- or vehicle-treated mice. E: Gene expression, evaluated by qPCR of Socs 3 and Lpl in the VAT of hepcidin- or vehicle-treated mice. White bars are the controls; black bars are the mice in the hepcidin administration group. Five mice per group were analyzed. Values are expressed as means $\pm \mathrm{SD} .{ }^{*} P<0.05$ versus controls at ANOVA. influences the physiology (or the differentiation process) of adipocytes, as also confirmed by gene expression analysis, which showed that the iron storage protein L-ferritin and the hormone hepcidin were among the most up-regulated genes. Intriguingly, VAT-IR was also paralleled by an increased expression and serum levels of resistin, an inflammatory adipokine with hyperglycemic action that decreases insulin sensitivity in mice. ${ }^{35}$ Increased resistin may in turn induce Socs3, an inhibitor of insulin signaling. ${ }^{25}$ Hepcidin, which was strongly up-regulated in the VAT of IED mice and whose serum levels were almost fivefold higher and in the range of DIOS, has also recently been shown to induce Socs 3 by activating intracellular signaling pathways, possibly contributing to VAT-IR. ${ }^{26,27,36}$

To investigate the possible role of altered mitochondrial iron metabolism in the pathogenesis of VAT insulin resistance associated with IED, we evaluated the expression of MitoNEET, a protein expressed on the outer mitochondrial membrane, that acts as a regulator of mitochondrial iron content and adipose tissue IR. ${ }^{28}$ However, we did not observe any difference in VAT expression of MitoNEET between IED and control mice, despite increased expression of mitochondrial antioxidant genes in IED mice (possibly in response to oxidative stress due to increased iron), thus suggesting that the effect of iron on VAT is not mediated by MitoNEET.

To dissect the relative contribution of iron overload and increased hepcidin in the biological alterations observed in the VAT of IED mice, we finally evaluated the effect of acute hepcidin administration. Despite no immediate effect on fasting glucose, triglyceride levels, and IR, hepcidin was associated with up-regulation of Socs 3 and down-regulation of Lpl in VAT, suggesting that in IED mice these alterations are independent of the metabolic milieu and represent a direct consequence of hepcidin up-regulation and establishing Lpl as a novel candidate transcriptional target of hepcidin.

After our manuscript was submitted, Gabrielsen et $\mathrm{al}^{37}$ independently reported that adipocyte iron regulates insulin sensitivity, as also confirmed by hyperinsulinemic clamp studies and adiponectin expression in mice in models of dietary supplementation and of ferroportin deletion in adipocytes. The observation that systemic iron overload is associated with IR, adipose tissue iron accumulation, and reduced adipose tissue mass is in line with our findings. At variance with our results, Gabrielsen et $\mathrm{al}^{37}$ reported that the effects of iron on adipose tissue mass are mediated by downregulation of adiponectin release. However, results were confounded by reduced fat mass in iron-overloaded mice, and the leanest iron-overloaded mice had to be excluded to detect an effect of iron on adiponectin levels. Therefore, the lack of down-regulation of adiponectin by IED in our model may be related to the more severe iron overload determined by our protocol, which was associated with the severely reduced adipose tissue mass. In addition, as we observed a similar phenotype, which was not explained by modulation of adiponectin levels, our results suggest that modulation of adiponectin transcription is unlikely to explain all of the 
metabolic disturbances induced by iron in the adipose tissue and at a systemic level, although we could not evaluate the effect of IED on different circulating adiponectin isoforms.

In conclusion, i) dietary iron overload with preserved upregulation of hepcidin is associated with increased fasting glucose and triglyceride levels, recapitulates the clinical features of DIOS, and could be exploited in further studies; ii) IED leads to the following alterations in VAT: iron accumulation, reduced mass, impaired insulin signaling, altered gene expression, and enhanced release of resistin; and iii) increased levels of hepcidin likely play a causal role in some of the gene expression alterations observed in the VAT of IED mice and possibly in other tissues. Evaluation of the effect of long-term manipulation of hepcidin, evaluation of the effect of iron in brown adipose tissue, and direct measurement of mitochondrial iron levels are required to better characterize the relative role of hepcidin and iron overload in the pathogenesis of the metabolic syndrome.

\section{Acknowledgments}

We thank Vittorio Borroni, Luca Benedan, Marta Milano, Fabio Ambrosetti, Marco Palazzo, Alessandra Alberghini, Benedetta Maria Motta, Flavio Caprioli (all from University of Milan), and Natascia Campostrini (University of Verona) for technical assistance and Ivana De Domenico (University of Utah, Salt Lake City, UT) for providing hepcidin-25.

\section{Supplemental Data}

Supplemental material for this article can be found at http://dx.doi.org/10.1016/j.ajpath.2013.02.019.

\section{References}

1. Dongiovanni P, Fracanzani AL, Fargion S, Valenti L: Iron in fatty liver and in the metabolic syndrome: a promising therapeutic target. J Hepatol 2011, 55:920-932

2. Valenti L, Fracanzani AL, Bugianesi E, Dongiovanni P, Galmozzi E, Vanni E, Canavesi E, Lattuada E, Roviaro G, Marchesini G, Fargion S: HFE genotype, parenchymal iron accumulation, and liver fibrosis in patients with nonalcoholic fatty liver disease. Gastroenterology 2010 , 138:905-912

3. Shah SV, Fonseca VA: Iron and diabetes revisited. Diabetes Care 2011, 34:1676-1677

4. Valenti L, Fracanzani AL, Dongiovanni P, Bugianesi E, Marchesini G, Manzini P, Vanni E, Fargion S: Iron depletion by phlebotomy improves insulin resistance in patients with nonalcoholic fatty liver disease and hyperferritinemia: evidence from a case-control study. Am J Gastroenterol 2007, 102:1251-1258

5. Pietrangelo A: Hemochromatosis: an endocrine liver disease. Hepatology 2007, 46:1291-1301

6. Nemeth E, Tuttle MS, Powelson J, Vaughn MB, Donovan A, Ward DM, Ganz T, Kaplan J: Hepcidin regulates cellular iron efflux by binding to ferroportin and inducing its internalization. Science 2004, 306:2090-2093

7. Aigner E, Theurl I, Theurl M, Lederer D, Haufe H, Dietze O, Strasser M, Datz C, Weiss G: Pathways underlying iron accumulation in human nonalcoholic fatty liver disease. Am J Clin Nutr 2008, 87: $1374-1383$

8. Fernandez-Real JM, Moreno JM, Chico B, Lopez-Bermejo A, Ricart W: Circulating visfatin is associated with parameters of iron metabolism in subjects with altered glucose tolerance. Diabetes Care 2007, 30:616-621

9. Green A, Basile R, Rumberger JM: Transferrin and iron induce insulin resistance of glucose transport in adipocytes. Metabolism 2006, 55: $1042-1045$

10. Wlazlo N, van Greevenbroek MMJ, Ferreira I, Jansen EHJM, Feskens EJ, van der Kallen CJH, Schalkwijk CG, Bravenboer B, Stehouwer CD: Iron metabolism is associated with adipocyte insulin resistance and plasma adiponectin. Diabetes Care 2012, [ePub ahead of print]

11. Huang J, Gabrielsen JS, Cooksey RC, Luo B, Boros LG, Jones DL, Jouihan HA, Soesanto Y, Knecht L, Hazel MW, Kushner JP, McClain DA: Increased glucose disposal and AMP-dependent kinase signaling in a mouse model of hemochromatosis. J Biol Chem 2007, 282:37501-37507

12. Huang J, Jones D, Luo B, Sanderson M, Soto J, Abel ED, Cooksey RC, McClain DA: Iron overload and diabetes risk: a shift from glucose to fatty acid oxidation and increased hepatic glucose production in a mouse model of hereditary hemochromatosis. Diabetes 2010, 60:80-87

13. Minamiyama Y, Takemura S, Kodai S, Shinkawa H, Tsukioka T, Ichikawa H, Naito Y, Yoshikawa T, Okada S: Iron restriction improves type 2 diabetes mellitus in Otsuka Long-Evans Tokushima fatty rats. Am J Physiol Endocrinol Metab 2010, 298:E1140-E1149

14. Cooksey RC, Jones D, Gabrielsen S, Huang J, Simcox JA, Luo B, Soesanto Y, Rienhoff H, Abel ED, McClain DA: Dietary iron restriction or iron chelation protects from diabetes and loss of beta-cell function in the obese (ob/ob lep-/-) mouse. Am J Physiol Endocrinol Metab 2010, 298:E1236-1243

15. Dongiovanni P, Valenti L, Ludovica Fracanzani A, Gatti S, Cairo G, Fargion S: Iron depletion by deferoxamine up-regulates glucose uptake and insulin signaling in hepatoma cells and in rat liver. Am J Pathol 2008, 172:738-747

16. Martinelli N, Traglia M, Campostrini N, Biino G, Corbella M, Sala C, Busti F, Masciullo C, Manna D, Previtali S, Castagna A, Pistis G, Olivieri O, Toniolo D, Camaschella C, Girelli D: Serum hepcidin levels in subjects with the metabolic syndrome: a population study. PLoS One 2012, (in press)

17. Troadec MB, Courselaud B, Detivaud L, Haziza-Pigeon C, Leroyer P, Brissot P, Loreal O: Iron overload promotes Cyclin D1 expression and alters cell cycle in mouse hepatocytes. J Hepatol 2006, 44:391-399

18. Svegliati-Baroni G, Candelaresi C, Saccomanno S, Ferretti G, Bachetti T, Marzioni M, De Minicis S, Nobili L, Salzano R, Omenetti A, Pacetti D, Sigmund S, Benedetti A, Casini A: A model of insulin resistance and nonalcoholic steatohepatitis in rats: role of peroxisome proliferator-activated receptor-alpha and n-3 polyunsaturated fatty acid treatment on liver injury. Am J Pathol 2006, 169: $846-860$

19. Wada T, Kenmochi H, Miyashita Y, Sasaki M, Ojima M, Sasahara M, Koya D, Tsuneki H, Sasaoka T: Spironolactone improves glucose and lipid metabolism by ameliorating hepatic steatosis and inflammation and suppressing enhanced gluconeogenesis induced by high-fat and high-fructose diet. Endocrinology 151:2040-2049

20. Rinella ME, Green RM: The methionine-choline deficient dietary model of steatohepatitis does not exhibit insulin resistance. J Hepatol 2004, 40:47-51

21. Zhang DL, Senecal T, Ghosh MC, Ollivierre-Wilson H, Tu T, Rouault TA: Hepcidin regulates ferroportin expression and intracellular iron homeostasis of erythroblasts. Blood 2011, 118:2868-2877

22. Recalcati S, Tacchini L, Alberghini A, Conte D, Cairo G: Oxidative stress-mediated down-regulation of rat hydroxyacid oxidase 1, a liverspecific peroxisomal enzyme. Hepatology 2003, 38:1159-1166

23. Cairo G, Tacchini L, Pogliaghi G, Anzon E, Tomasi A, BernelliZazzera A: Induction of ferritin synthesis by oxidative stress: 
transcriptional and post-transcriptional regulation by expansion of the "free" iron pool. J Biol Chem 1995, 270:700-703

24. Girelli D, Trombini P, Busti F, Campostrini N, Sandri M, Pelucchi S, Westerman M, Ganz T, Nemeth E, Piperno A, Camaschella C: A time course of hepcidin response to iron challenge in patients with HFE and TFR2 hemochromatosis. Haematologica 96:500-506

25. Steppan CM, Wang J, Whiteman EL, Birnbaum MJ, Lazar MA: Activation of SOCS-3 by resistin. Mol Cell Biol 2005, 25:1569-1575

26. De Domenico I, Zhang TY, Koening CL, Branch RW, London N, Lo E, Daynes RA, Kushner JP, Li D, Ward DM, Kaplan J: Hepcidin mediates transcriptional changes that modulate acute cytokine-induced inflammatory responses in mice. J Clin Invest 2010, 120:2395-2405

27. Ueki K, Kondo T, Tseng YH, Kahn CR: Central role of suppressors of cytokine signaling proteins in hepatic steatosis, insulin resistance, and the metabolic syndrome in the mouse. Proc Natl Acad Sci U S A 2004, 101:10422-10427

28. Kusminski CM, Holland WL, Sun K, Park J, Spurgin SB, Lin Y, Askew GR, Simcox JA, McClain DA, Li C, Scherer PE: MitoNEETdriven alterations in adipocyte mitochondrial activity reveal a crucial adaptive process that preserves insulin sensitivity in obesity. Nat Med 2012, 18:1539-1549

29. Tan TC, Crawford DH, Jaskowski LA, Murphy TM, Heritage ML, Subramaniam VN, Clouston AD, Anderson GJ, Fletcher LM: Altered lipid metabolism in Hfe-knockout mice promotes severe NAFLD and early fibrosis. Am J Physiol Gastrointest Liver Physiol 2011, 301: G865-G876
30. Nelson JE, Wilson L, Brunt EM, Yeh MM, Kleiner DE, UnalpArida A, Kowdley KV: Relationship between the pattern of hepatic iron deposition and histological severity in nonalcoholic fatty liver disease. Hepatology 2011, 53:448-457

31. Li X, Monks B, Ge Q, Birnbaum MJ: Akt/PKB regulates hepatic metabolism by directly inhibiting PGC-1alpha transcription coactivator. Nature 2007, 447:1012-1016

32. Nandi A, Kitamura Y, Kahn CR, Accili D: Mouse models of insulin resistance. Physiol Rev 2004, 84:623-647

33. Jo J, Gavrilova O, Pack S, Jou W, Mullen S, Sumner AE, Cushman SW, Periwal V: Hypertrophy and/or hyperplasia: dynamics of adipose tissue growth. PLoS Comput Biol 2009, 5:e1000324

34. McLaughlin T, Sherman A, Tsao P, Gonzalez O, Yee G, Lamendola C, Reaven GM, Cushman SW: Enhanced proportion of small adipose cells in insulin-resistant vs insulin-sensitive obese individuals implicates impaired adipogenesis. Diabetologia 2007, 50:1707-1715

35. Filkova M, Haluzik M, Gay S, Senolt L: The role of resistin as a regulator of inflammation: implications for various human pathologies. Clin Immunol 2009, 133:157-170

36. Sabio G, Das M, Mora A, Zhang Z, Jun JY, Ko HJ, Barrett T, Kim JK, Davis RJ: A stress signaling pathway in adipose tissue regulates hepatic insulin resistance. Science 2008, 322:1539-1543

37. Gabrielsen JS, Gao Y, Simcox JA, Huang J, Thorup D, Jones D, Cooksey RC, Gabrielsen D, Adams TD, Hunt SC, Hopkins PN, Cefalu WT, McClain DA: Adipocyte iron regulates adiponectin and insulin sensitivity. J Clin Invest 2012, 122:3529-3540 